\title{
Multivariate contraction mapping principle in Menger probabilistic metric spaces
}

\author{
Jinyu Guan ${ }^{a}$, Yanxia Tang ${ }^{a}$, Yongchun Xu ${ }^{a}$, Yongfu Su ${ }^{b, *}$ \\ ${ }^{a}$ Department of Mathematics, College of Science, Hebei North University, Zhangjiakou 075000, China. \\ ${ }^{b}$ Department of Mathematics, Tianjin Polytechnic University, Tianjin 300387, China.
}

Communicated by C. Zaharia

\begin{abstract}
The purpose of this paper is to prove the multivariate contraction mapping principle of $\mathrm{N}$-variables mappings in Menger probabilistic metric spaces. In order to get the multivariate contraction mapping principle, the product spaces of Menger probabilistic metric spaces are subtly defined which is used as an important method for the expected results. Meanwhile, the relative iterative algorithm of the multivariate fixed point is established. The results of this paper improve and extend the contraction mapping principle of single variable mappings in the probabilistic metric spaces. (C)2017 All rights reserved.
\end{abstract}

Keywords: Contraction mapping principle, probabilistic metric spaces, product spaces, multivariate fixed point. 2010 MSC: 46S10, 47H10.

\section{Introduction and preliminaries}

It is, in some cases, more appropriate to work with an average of several measurements as a measure to evaluate the distance between two points. In lines of this approach, Menger $[16,17]$ introduced the notion of probabilistic metric spaces as a generalization of metric spaces. Actually, Menger replaced the distance function $d(x, y)$ with a distribution function $F_{x, y}: X \times X \rightarrow R$, in such a way that, for any number $t$, the value $F_{x, y}(t)$ describes the probability that the distance between $x$ and $y$ is less than $t$. Later, the study of probabilistic metric spaces received a new impulse after the seminal work of Schweizer and Sklar [19]. The theory of probabilistic metric spaces is also very important in random functional analysis, random differential equation theory and the mathematics of fractals (see [1]). Sehgal and Bharucha-Reid [20, 21] established fixed point theorems in probabilistic metric spaces (for short, PM-spaces). Indeed, by using the notion of probabilistic q-contraction, they proved a unique fixed point result, which is an extension of the celebrated Banach contraction principle. For the interested reader, a comprehensive study of fixed point theory in the probabilistic metric setting can be found in the book of Hadzić and Pap [12], see also [25] for further discussion on generalizations of metric fixed point theory. Recently, Choudhury and Das [4] gave

\footnotetext{
*Corresponding author

Email addresses: guanj inyu2010@163.com (Jinyu Guan), sutang2016@163.com (Yanxia Tang), hbxuyongchun@163.com (Yongchun $\mathrm{Xu}$ ), tj suyongfu@163.com (Yongfu Su)

doi:10.22436/jnsa.010.09.17
} 
a generalized unique fixed point theorem by using an altering distance function, which was originally introduced by Khan et al. [13]. For other results in this direction, we refer to [2,4-11, 18]. In particular, Dutta et al. [10] defined nonlinear generalized contractive type mappings involving altering distances (say, $\psi$-contractive mappings) in Menger PM-spaces and proved their theorem for such kind of mappings in the setting of G-complete Menger PM-spaces. On contributing to this study, In 2015, M. Kutbi et al. [14] weakened the notion of $\psi$-contractive mapping and established some fixed point theorems in G-complete and M-complete Menger PM-spaces, besides discussing some related results and illustrative examples. In 2014, Su and Zhang [24] proved some fixed point and best proximity point theorems for contractions in a class of probabilistic metric spaces. In 2015, Su et al. [22] proved the generalized contraction mapping principle and generalized best proximity point theorems in probabilistic metric spaces.

Recently, the multivariate fixed point problems (or a fixed point of order N, see [15]) were studied by several authors. In 2016, Su et al. [23] proved some multivariate fixed point theorems by using a clever way.

The purpose of this paper is to prove the multivariate contraction mapping principle of $\mathrm{N}$-variables mappings in Menger probabilistic metric spaces. In order to get the multivariate contraction mapping principle, the product spaces of Menger probabilistic metric spaces are subtly defined which is used as an important method for the expected results. Meanwhile, the relative iterative algorithm of the multivariate fixed point is established. The results of this paper improve and extend the contraction mapping principle of single variable mappings in the probabilistic metric spaces.

Next we shall recall some well-known definitions and results in the theory of probabilistic metric spaces which are used later on this paper. For more details, we refer the reader to $[6,7,12]$.

Definition 1.1. A triangular norm (shorter $T$-norm) is a binary operation $T$ on $[0,1]$ which satisfies the following conditions:

(a) $\mathrm{T}$ is associative and commutative;

(b) $\mathrm{T}$ is continuous;

(c) $\mathrm{T}(\mathrm{a}, 1)=a$ for all $\mathrm{a} \in[0,1]$;

(d) $T(a, b) \leqslant T(c, d)$ whenever $a \leqslant c$ and $b \leqslant d$ for each $a, b, c, d \in[0,1]$.

The following are the four basic T-norms:

- $\mathrm{T}_{1}(\mathrm{a}, \mathrm{b})=\max (\mathrm{a}+\mathrm{b}-1,0)$;

- $T_{2}(a, b)=a \cdot b$;

- $\mathrm{T}_{3}(a, b)=\left\{\begin{array}{cc}\frac{a b}{a+b-a b}, & a b \neq 0, \\ 0, & a b=0 ;\end{array}\right.$

- $\mathrm{T}_{4}(\mathrm{a}, \mathrm{b})=\min (\mathrm{a}, \mathrm{b})$.

It is easy to check the above four T-norms have the following relations:

$$
T_{1}(a, b) \leqslant T_{2}(a, b) \leqslant T_{3}(a, b) \leqslant T_{4}(a, b)
$$

for any $a, b \in[0,1]$.

Definition 1.2. A function $F(t):(-\infty,+\infty) \rightarrow[0,1]$ is called a distance distribution function if it is nondecreasing and left-continuous with $\lim _{t \rightarrow-\infty} F(t)=0, \lim _{t \rightarrow+\infty} F(t)=1$ and $F(0)=0$. The set of all distance distribution functions is denoted by $\mathrm{D}^{+}$. A special Menger distance distribution function is given by

$$
H(t)= \begin{cases}0, & t \leqslant 0 \\ 1, & t>0\end{cases}
$$


Definition 1.3. A probabilistic metric space is a pair $(E, F)$, where $E$ is a nonempty set, $F$ is a mapping from $E \times E$ into $D^{+}$such that, if $F_{x, y}$ denotes the value of $F$ at the pair $(x, y)$, the following conditions hold:

(PM-1) $F_{x, y}(t)=H(t)$ if and only if $x=y$;

(PM-2) $F_{x, y}(t)=F_{y, x}(t)$ for all $x, y \in E$ and $t \in(-\infty,+\infty)$;

(PM-3) $F_{x, z}(t)=1 \quad F_{z, y}(s)=1$ implies $F_{x, y}(t+s)=1$,

for all $x, y, z \in E$ and $t \in(-\infty,+\infty)$.

Definition 1.4. A Menger probabilistic metric space is a triple $(E, F, T)$ where $E$ is a nonempty set, $T$ is a continuous t-norm and $F$ is a mapping from $E \times E$ into $D^{+}$such that if $F_{x, y}$ denotes the value of $F$ at the pair $(x, y)$, the following conditions hold:

(MPM-1) $F_{x, y}(t)=H(t)$ if and only if $x=y$;

(MPM-2) $F_{x, y}(t)=F_{y, x}(t)$ for all $x, y \in E$ and $t \in(-\infty,+\infty)$;

(MPM-3) $F_{x, y}(t+s) \geqslant T\left(F_{x, z}(t), F_{z, y}(s)\right)$ for all $x, y, z \in E$ and $t>0, s>0$.

Definition 1.5. Let $(E, F, T)$ be a probabilistic metric space.

(1) A sequence $\left\{x_{n}\right\}$ in $E$ is said to converge to $x \in E$, if for any given $\varepsilon>0$ and $\lambda>0$, there must exist a positive integer $N=N(\varepsilon, \lambda)$ such that $F_{x_{n}, x}(\varepsilon)>1-\lambda$ whenever $n>N$.

(2) A sequence, $\left\{x_{n}\right\}$ in $E$ is called a Cauchy sequence if for any $\varepsilon>0$ and $\lambda>0$, there must exist a positive integer $N=N(\varepsilon, \lambda)$ such that $F_{x_{n}, x_{m}}(\varepsilon)>1-\lambda$, whenever $n, m>N$.

(3) $(E, F, T)$ is said to be complete, if each Cauchy sequence in $E$ converges to some point in $E$.

\section{Main results}

In this section, we firstly give the concept of multiply probabilistic metric function which will play an important role in this article.

Definition 2.1. Let $T$ be a given T-norm. A multiply probabilistic metric function $P_{T}\left(a_{1}, a_{2}, \cdots, a_{N}\right)$ is a continuous $\mathrm{N}$ variables real function with the domain

$$
\left\{\left(a_{1}, a_{2}, \cdots, a_{N}\right) \in R^{N}: 0 \leqslant a_{i} \leqslant 1, i \in\{1,2,3, \cdots, N\},\right.
$$

and the range $[0,1]$ which satisfies the following conditions:

(1) $P_{\mathrm{T}}\left(a_{1}, a_{2}, \cdots, a_{N}\right)$ is non-decreasing for each variable $a_{i}, i \in\{1,2,3, \cdots, N\}$;

(2) $P_{T}\left(T\left(a_{1}, b_{1}\right), T\left(a_{2}, b_{2}\right), \cdots, T\left(a_{N}, b_{N}\right)\right) \geqslant T\left(P_{T}\left(a_{1}, a_{2}, \cdots, a_{N}\right), P_{T}\left(b_{1}, b_{2}, \cdots, b_{N}\right)\right)$;

(3) $P_{\mathrm{T}}(a, a, \cdots, a)=a$;

(4) $P_{\mathrm{T}}\left(a_{1}, a_{2}, \cdots, a_{N}\right) \rightarrow 1 \Leftrightarrow a_{i} \rightarrow 1, i \in\{1,2,3, \cdots, N\}$

for all $a_{i}, b_{i}, a \in \mathbb{R}, i \in\{1,2,3, \cdots, N\}$, where $\mathbb{R}$ denotes the set of all real numbers.

Example 2.2. For $T(a, b)=\max \{a+b-1,0\}$, the $N$ variables real function

$$
P_{T}\left(a_{1}, a_{2}, a_{3}, \ldots, a_{N}\right)=\frac{1}{N} \sum_{i=1}^{N} a_{i},
$$

is a multiply probabilistic metric function. The above conditions (1), (3), (4) are obvious. Next, we check 
the condition (2).

$$
\begin{aligned}
P_{T}\left(T\left(a_{1}, b_{1}\right), T\left(a_{2}, b_{2}\right), \cdots, \Delta\left(a_{N}, b_{N}\right)\right) & =\frac{1}{N} \sum_{i=1}^{N} T\left(a_{i}, b_{i}\right) \\
& =\frac{1}{N} \sum_{i=1}^{N} \max \left\{a_{i}+b_{i}-1,0\right\} \\
& \geqslant \frac{1}{N} \max \left\{\sum_{i=1}^{N}\left(a_{i}+b_{i}-1\right), 0\right\} \\
& =\max \left\{\frac{1}{N} \sum_{i=1}^{N} a_{i}+\frac{1}{N} \sum_{i=1}^{N} b_{i}-1,0\right\} \\
& =T\left(P_{T}\left(a_{1}, a_{2}, \cdots, a_{N}\right), P_{T}\left(b_{1}, b_{2}, \cdots, b_{N}\right)\right) .
\end{aligned}
$$

Example 2.3. For $T(a, b)=\max \{a+b-1,0\}$, the $N$ variables real function

$$
P_{\mathrm{T}}\left(a_{1}, a_{2}, a_{3}, \cdots, a_{N}\right)=\sum_{i=1}^{N} \lambda_{i} a_{i}
$$

is a multiply probabilistic metric function, where $\lambda_{i}, i=1,2,3, \cdots, N$ are constants and

$$
0<\lambda_{i}<1, \sum_{i=1}^{N} \lambda_{i}=1 .
$$

The above conditions (1), (3), (4) are obvious. Next, we check the condition (2).

$$
\begin{aligned}
P_{T}\left(T\left(a_{1}, b_{1}\right), T\left(a_{2}, b_{2}\right), \cdots, T\left(a_{N}, b_{N}\right)\right) & =\sum_{i=1}^{N} \lambda_{i} T\left(a_{i}, b_{i}\right) \\
& =\sum_{i=1}^{N} \lambda_{i} \max \left\{a_{i}+b_{i}-1,0\right\} \\
& \geqslant \max \left\{\sum_{i=1}^{N} \lambda_{i}\left(a_{i}+b_{i}-1\right), 0\right\} \\
& =\max \left\{\sum_{i=1}^{N} \lambda_{i} a_{i}+\sum_{i=1}^{N} \lambda_{i} b_{i}-1,0\right\} \\
& =T\left(P_{T}\left(a_{1}, a_{2}, \cdots, a_{N}\right), P_{T}\left(b_{1}, b_{2}, \cdots, b_{N}\right)\right) .
\end{aligned}
$$

Definition 2.4. Let $(E, F)$ be a probabilistic metric space, $T: E^{N} \rightarrow E$ be an $N$-variables mapping, an element $p \in E$ is called a multivariate fixed point if

$$
p=T(p, p, \cdots, p) .
$$

The following theorem is one of the main results which will play an important role.

Theorem 2.5. Let $(\mathrm{E}, \mathrm{F}, \mathrm{T})$ be a Menger probabilistic metric space. Let $\mathrm{E}^{\mathrm{N}}$ be the Cartesian product of $\mathrm{E}$ and

$$
D_{x, y}(t)=P_{T}\left(F_{x_{1}, y_{1}}(t), F_{x_{2}, y_{2}}(t), \cdots, F_{x_{N}, y_{N}}(t)\right)
$$

for all $\mathrm{x}=\left(\mathrm{x}_{1}, \mathrm{x}_{2}, \cdots, \mathrm{x}_{\mathrm{N}}\right), \mathrm{y}=\left(\mathrm{y}_{1}, \mathrm{y}_{2}, \cdots, \mathrm{y}_{\mathrm{N}}\right) \in \mathrm{E}^{\mathrm{N}}$, where $\mathrm{P}_{\mathrm{T}}$ is a multiply probabilistic metric function. Then $\left(\mathrm{E}^{\mathrm{N}}, \mathrm{D}, \mathrm{T}\right)$ is a Menger probabilistic metric space. Further, $\left(\mathrm{E}^{\mathrm{N}}, \mathrm{D}, \mathrm{T}\right)$ is complete provided $(\mathrm{E}, \mathrm{F}, \mathrm{T})$ is complete. 
Proof. From the continuity of multiply probabilistic metric function and the conditions (3), (4) of Definition 2.1, we know that $D_{x, y}(t)$ is a distance distribution function for all $x, y \in E^{N}$. That is, $D_{x, y}(t)$ is nondecreasing and left-continuous with $\lim _{t \rightarrow-\infty} D_{x, y}(t)=0, \lim _{t \rightarrow+\infty} D_{x, y}(t)=1$ and $D_{x, y}(0)=0$ for all $x, y \in E^{N}$. Next, we check the conditions (MPM-1)-(MPM-3) of Definition 1.4. The conditions (MPM-1) and (MPM-2) are obvious. Now, we check the condition (MPM-3). For all

$$
x=\left(x_{1}, x_{2}, \cdots, x_{N}\right), y=\left(y_{1}, y_{2}, \cdots, y_{N}\right), z=\left(z_{1}, z_{2}, \cdots, z_{N}\right) \in E^{N},
$$

and $t, s>0$, from the condition (2) of Definition 2.1, we have that

$$
\begin{aligned}
& D_{x, y}(t+s)=P_{T}\left(F_{x_{1}, y_{1}}(t+s), F_{x_{2}, y_{2}}(t+s), \cdots, F_{x_{N}, y_{N}}(t+s)\right) \\
& \geqslant P_{T}\left(T\left(F_{x_{1}, z_{1}}(t), F_{z_{1}, y_{1}}(s)\right), T\left(F_{x_{2}, z_{2}}(t), F_{z_{2}, y_{2}}(s)\right), \cdots, T\left(F_{x_{N}, z_{N}}(t), F_{z_{N}, y_{N}}(s)\right)\right) \\
& \geqslant T\left(P_{T}\left(F_{x_{1}, z_{1}}(t), F_{x_{2}, z_{2}}(t), \cdots, F_{x_{N}, z_{N}}(t)\right), P_{T}\left(F_{z_{1}, y_{1}}(s), F_{z_{2}, y_{2}}(s), \cdots, F_{z_{N}, y_{N}}(s)\right)\right) \\
& =T\left(D_{x, z}(t), D_{z, y}(s)\right) \text {. }
\end{aligned}
$$

Hence $\left(E^{N}, D, T\right)$ is a Menger probabilistic metric space.

Let $\left\{x_{n}=\left(x_{1, n}, x_{2, n}, \cdots, x_{N, n}\right)\right\} \in\left(E^{N}, D, T\right)$ be a Cauchy sequence. That is,

$$
\lim _{n, m \rightarrow+\infty} D_{x_{n}, x_{m}}(t)=1, \quad \forall t>0 .
$$

This is equivalent to

$$
\lim _{n, m \rightarrow+\infty} F_{x_{i, n}, x_{i, m}}(t)=1, \quad \forall t>0, \quad \forall i=1,2,3, \cdots, N .
$$

Since $(E, F, T)$ is complete, there exist $x_{1}, x_{2}, \cdots, x_{N} \in E$ such that $x_{i, n}$ converges to $x_{i}$ for all $i=$ $1,2,3, \cdots, N$. That is

$$
\lim _{n \rightarrow+\infty} F_{x_{i, n}, x_{i}}(t)=1, \quad \forall t>0, \forall i=1,2,3, \cdots, N,
$$

which implies that

$$
\lim _{n \rightarrow+\infty} D_{x_{n}, x}(t)=1, \quad \forall t>0,
$$

where $x=\left(x_{1}, x_{2}, \cdots, x_{N}\right) \in E^{N}$. Hence $\left(E^{N}, D, T\right)$ is complete. This completes the proof.

Corollary 2.6. Let $\left(\mathrm{E}, \mathrm{F}, \mathrm{T}_{1}\right)$ be a Menger probabilistic metric space, where $\mathrm{T}_{1}(\mathrm{a}, \mathrm{b})=\max \{\mathrm{a}+\mathrm{b}-1,0\}$. Let $\mathrm{E}^{\mathrm{N}}$ be the Cartesian product of $\mathrm{E}$ and

$$
D_{x, y}(t)=\sum_{i=1}^{N} \lambda_{i} F_{x_{i}, y_{i}}(t)
$$

for all $x=\left(x_{1}, x_{2}, \cdots, x_{N}\right), y=\left(y_{1}, y_{2}, \cdots, y_{N}\right) \in E^{N}$, where $\lambda_{i}, i=1,2,3, \cdots, N$ are constants and $0<\lambda_{i}<$ $1, \sum_{i=1}^{N} \lambda_{i}=1$. Then $\left(E^{N}, D, T_{1}\right)$ is a Menger probabilistic metric space. Further, $\left(E^{N}, D, T_{1}\right)$ is complete provided $\left(\mathrm{E}, \mathrm{F}, \mathrm{T}_{1}\right)$ is complete.

In special, we have the following result.

Corollary 2.7. Let $\left(\mathrm{E}, \mathrm{F}, \mathrm{T}_{1}\right)$ be a Menger probabilistic metric space, where $\mathrm{T}_{1}(\mathrm{a}, \mathrm{b})=\max \{\mathrm{a}+\mathrm{b}-1,0\}$. Let $\mathrm{E}^{\mathrm{N}}$ be the Cartesian product of $\mathrm{E}$ and

$$
D_{x, y}(t)=\frac{1}{N} \sum_{i=1}^{N} F_{x_{i}, y_{i}}(t)
$$

for all $\mathrm{x}=\left(\mathrm{x}_{1}, \mathrm{x}_{2}, \cdots, \mathrm{x}_{\mathrm{N}}\right), \mathrm{y}=\left(\mathrm{y}_{1}, \mathrm{y}_{2}, \cdots, \mathrm{y}_{\mathrm{N}}\right) \in \mathrm{E}^{\mathrm{N}}$. Then $\left(\mathrm{E}^{\mathrm{N}}, \mathrm{D}, \mathrm{T}_{1}\right)$ is a Menger probabilistic metric space. Further, $\left(\mathrm{E}^{\mathrm{N}}, \mathrm{D}, \mathrm{T}_{1}\right)$ is complete provided $\left(\mathrm{E}, \mathrm{F}, \mathrm{T}_{1}\right)$ is complete.

In 1972, Sehgal and Bharucha-Ried [21] proved a unique fixed point result, which is an extension of the celebrated Banach contraction mapping principle 
Lemma 2.8 ([21]). Let (E, F, T) be a complete Menger probabilistic metric space with a continuous T-norm $\mathrm{T}$. Let $\mathrm{T}: \mathrm{E} \rightarrow \mathrm{E}$ be a mapping satisfying the following condition

$$
F_{T x, T y}(t) \geqslant F_{x, y}\left(\frac{t}{h}\right)
$$

for every $\mathrm{x}, \mathrm{y} \in \mathrm{E}$ and $\mathrm{t}>0$, where $\mathrm{h} \in(0,1)$ is a constant. Then either

(i) Thas a unique fixed point; or

(ii) for every $\mathrm{p}_{0} \in \mathrm{E}, \sup \left\{\mathrm{G}_{\mathrm{p}_{0}}(\mathrm{t}): \mathrm{t} \in \mathrm{R}\right\}<1$, where

$$
\mathrm{G}_{\mathrm{p}_{0}}(\mathrm{t})=\inf \left\{\mathrm{F}_{\mathrm{p}_{0}, \mathrm{p}_{\mathrm{n}}}(\mathrm{t})\right\}, \quad \mathrm{p}_{\mathrm{n}}=\mathrm{Tp}_{\mathrm{n}-1}, \quad \mathrm{n}=1,2,3, \cdots .
$$

Theorem 2.9 ([21]). Let $(\mathrm{E}, \mathrm{F}, \mathrm{T})$ be a complete Menger probabilistic metric space with a continuous T-norm $\mathrm{T}(\mathrm{a}, \mathrm{b})=\min \{\mathrm{a}, \mathrm{b}\}$. Let $\mathrm{T}: \mathrm{E} \rightarrow \mathrm{E}$ be a mapping satisfying the following condition:

$$
F_{T x, T y}(t) \geqslant F_{x, y}\left(\frac{t}{h}\right)
$$

for every $\mathrm{x}, \mathrm{y} \in \mathrm{E}$ and $\mathrm{t}>0$, where $\mathrm{h} \in(0,1)$ is a constant. Then $\mathrm{T}$ has a unique fixed point.

In what follows, we prove the following theorems, which generalize the result of Sehgal and BharuchaRied [21].

Theorem 2.10. Let $(\mathrm{E}, \mathrm{F}, \mathrm{T})$ be a complete Menger probabilistic metric space with a continuous T-norm $\mathrm{T}$. Let $\mathrm{T}: \mathrm{E}^{\mathrm{N}} \rightarrow \mathrm{E}$ be an $\mathrm{N}$-variables mapping satisfying the following condition:

$$
F_{T x, T y}(t) \geqslant P_{T}\left(F_{x_{1}, y_{1}}\left(\frac{t}{h}\right), F_{x_{2}, y_{2}}\left(\frac{t}{h}\right), \cdots, F_{x_{N}, y_{N}}\left(\frac{t}{h}\right)\right)
$$

for every

$$
x=\left(x_{1}, x_{2}, \cdots, x_{N}\right) \in X^{N}, \quad y=\left(y_{1}, y_{2}, \cdots, y_{N}\right) \in X^{N},
$$

and $\mathrm{t}>0$, where $\mathrm{h} \in(0,1)$ is a constant and $\mathrm{P}_{\mathrm{T}}$ is a probabilistic multiply metric function. Assume there exists $\mathrm{p}_{0} \in \mathrm{E}$ such that $\sup \left\{\mathrm{G}_{\mathrm{p}_{0}}(\mathrm{t}): \mathrm{t} \in \mathrm{R}\right\}=1$, where

$$
\begin{aligned}
\mathrm{G}_{\mathrm{p}_{0}}(\mathrm{t}) & =\inf \left\{\mathrm{F}_{\mathrm{p}_{0}, \mathrm{p}_{\mathrm{n}}}(\mathrm{t})\right\}, \quad \mathrm{n}=1,2,3, \cdots, \\
\mathrm{p}_{1} & =\mathrm{T}\left(\mathrm{p}_{0}, \mathrm{p}_{0}, \cdots, \mathrm{p}_{0}\right), \\
\mathrm{p}_{2} & =\mathrm{T}\left(\mathrm{p}_{1}, \mathrm{p}_{1}, \cdots, \mathrm{p}_{1}\right), \\
\mathrm{p}_{3} & =\mathrm{T}\left(\mathrm{p}_{2}, \mathrm{p}_{2}, \cdots, \mathrm{p}_{2}\right), \\
& \vdots \\
\mathrm{p}_{\mathrm{n}} & =\mathrm{T}\left(\mathrm{p}_{\mathrm{n}-1}, \mathrm{p}_{\mathrm{n}-1}, \cdots, \mathrm{p}_{\mathrm{n}-1}\right),
\end{aligned}
$$

Then Thas a unique multivariate fixed point.

Proof. Let $\mathrm{E}^{\mathrm{N}}$ be the Cartesian product of $\mathrm{E}$ and

$$
D_{x, y}(t)=P_{T}\left(F_{x_{1}, y_{1}}(t), F_{x_{2}, y_{2}}(t), \cdots, F_{x_{N}, y_{N}}(t)\right)
$$

for all $x=\left(x_{1}, x_{2}, \cdots, x_{N}\right), y=\left(y_{1}, y_{2}, \cdots, y_{N}\right) \in E^{N}$, where $P_{T}$ is a multiply probabilistic metric function. Then $\left(E^{N}, D, T\right)$ is a complete Menger probabilistic metric space. Let $T^{*}: E^{N} \rightarrow E^{N}$ be defined by

$$
\mathrm{T}^{*}:\left(\mathrm{x}_{1}, \mathrm{x}_{2}, \cdots, \mathrm{x}_{\mathrm{N}}\right) \mapsto(\mathrm{T} x, \mathrm{~T} x, \cdots, \mathrm{T} x)
$$


for all $x=\left(x_{1}, x_{2}, \cdots, x_{N}\right) \in E^{N}$. In this case, we have that

$$
\begin{aligned}
\mathrm{D}_{\mathrm{T}^{*} x, T^{*} y}(\mathrm{t}) & =\mathrm{P}_{\mathrm{T}}\left(\mathrm{F}_{\mathrm{T} x, T y}(\mathrm{t}), \mathrm{F}_{\mathrm{T} x, T y}(\mathrm{t}), \ldots, \mathrm{F}_{\mathrm{T} x, T y}(\mathrm{t})\right) \\
& =\mathrm{F}_{\mathrm{T} x, T y}(\mathrm{t}) \\
& \geqslant \mathrm{P}_{\mathrm{T}}\left(\mathrm{F}_{x_{1}, y_{1}}\left(\frac{\mathrm{t}}{\mathrm{h}}\right), \mathrm{F}_{x_{2}, y_{2}}\left(\frac{\mathrm{t}}{\mathrm{h}}\right), \cdots, \mathrm{F}_{x_{N}, y_{N}}\left(\frac{t}{h}\right)\right) \\
& =\mathrm{D}_{x, y}\left(\frac{\mathrm{t}}{\mathrm{h}}\right)
\end{aligned}
$$

for all $x=\left(x_{1}, x_{2}, \cdots, x_{N}\right) \in E^{N}, y=\left(y_{1}, y_{2}, \cdots, y_{N}\right) \in E^{N}$. By using Lemma 2.8, then either

(i) $\mathrm{T}^{*}$ has a unique fixed point $x^{*} \in \mathrm{E}^{\mathrm{N}}$; or

(ii) for every $P_{0} \in E^{N}, \sup \left\{G_{P_{0}}(t): t \in R\right\}<1$, where

$$
G_{P_{0}}(t)=\inf \left\{D_{P_{0}, P_{n}}(t)\right\}, \quad P_{n}=T^{*} P_{n-1}, \quad n=1,2,3, \cdots .
$$

In the case (i): There exists a unique $x^{*}=\left(x_{1}^{*}, x_{2}^{*}, \cdots, x_{N}^{*}\right) \in E^{N}$ such that

$$
\begin{aligned}
\mathrm{T}^{*} x^{*} & =\left(T x^{*}, T x^{*}, \cdots, T x^{*}\right) \\
& =\left(x_{1}^{*}, x_{2}^{*}, \cdots, x_{N}^{*}\right) .
\end{aligned}
$$

This implies that $x_{1}^{*}=x_{2}^{*}=\cdots=x_{N}^{*}$ and $x_{1}^{*}=T\left(x_{1}^{*}, x_{1}^{*}, \cdots, x_{1}^{*}\right)$, hence $T$ has a unique multivariate fixed point $x_{1}^{*}$.

In the case (ii): We take $P_{0}=\left(p_{0}, p_{0}, \cdots, p_{0}\right) \in E^{N}$, then

$$
\begin{aligned}
\mathrm{D}_{\mathrm{P}_{0}, \mathrm{P}_{\mathfrak{n}}}(\mathrm{t}) & =\mathrm{P}_{\mathrm{T}}\left(\mathrm{F}_{\mathrm{p}_{0}, \mathrm{p}_{\mathfrak{n}}}(\mathrm{t}), \mathrm{F}_{\mathrm{p}_{0}, \mathrm{p}_{\mathfrak{n}}}(\mathrm{t}), \cdots, \mathrm{F}_{\mathrm{p}_{0}, \mathrm{p}_{\mathfrak{n}}}(\mathrm{t})\right) \\
& =\mathrm{F}_{\mathrm{p}_{0}, \mathrm{p}_{\mathfrak{n}}}(\mathrm{t}) .
\end{aligned}
$$

Hence

$$
\begin{aligned}
\mathrm{G}_{\mathrm{P}_{0}}(\mathrm{t}) & =\inf \left\{\mathrm{D}_{\mathrm{P}_{0}, \mathrm{P}_{\mathfrak{n}}}(\mathrm{t})\right\} \\
& =\inf \left\{\mathrm{F}_{\mathrm{p}_{0}, \mathrm{p}_{\mathfrak{n}}}(\mathrm{t})\right\} \\
& =\mathrm{G}_{\mathrm{p}_{0}}(\mathrm{t}) .
\end{aligned}
$$

From the condition of Theorem 2.10, we know $\sup \left\{\mathrm{G}_{\mathrm{P}_{0}}(\mathrm{t}): \mathrm{t} \in \mathrm{R}\right\}=1$. This is a contradiction. This completes the proof.

Theorem 2.11. Let $(\mathrm{E}, \mathrm{F}, \mathrm{T})$ be a complete Menger probabilistic metric space with a continuous $\mathrm{T}-n \mathrm{n} m \mathrm{~T}(\mathrm{a}, \mathrm{b})=$ $\min \{\mathrm{a}, \mathrm{b}\}$. Let $\mathrm{T}: \mathrm{E}^{\mathrm{N}} \rightarrow \mathrm{E}$ be an $\mathrm{N}$-variables mapping satisfying the following condition:

$$
F_{T x, T y}(t) \geqslant P_{T}\left(F_{x_{1}, y_{1}}\left(\frac{t}{h}\right), F_{x_{2}, y_{2}}\left(\frac{t}{h}\right), \cdots, F_{x_{N}, y_{N}}\left(\frac{t}{h}\right)\right),
$$

for every

$$
x=\left(x_{1}, x_{2}, \cdots, x_{N}\right) \in X^{N}, \quad y=\left(y_{1}, y_{2}, \cdots, y_{N}\right) \in X^{N},
$$

and $\mathrm{t}>0$, where $\mathrm{h} \in(0,1)$ is a constant and $\mathrm{P}_{\mathrm{T}}$ is a probabilistic multiply metric function. Then $\mathrm{T}$ has a unique multivariate fixed point.

Proof. Let $\mathrm{E}^{\mathrm{N}}$ be the Cartesian product of $\mathrm{E}$ and

$$
D_{x, y}(t)=P_{T}\left(F_{x_{1}, y_{1}}(t), F_{x_{2}, y_{2}}(t), \cdots, F_{x_{N}, y_{N}}(t)\right)
$$

for all $x=\left(x_{1}, x_{2}, \cdots, x_{N}\right), y=\left(y_{1}, y_{2}, \cdots, y_{N}\right) \in E^{N}$, where $P_{T}$ is a multiply probabilistic metric function. 
Then $\left(E^{N}, D, T\right)$ is a complete Menger probabilistic metric space with the $T$-norm $T(a, b)=\min \{a, b\}$. Let $\mathrm{T}^{*}: \mathrm{E}^{\mathrm{N}} \rightarrow \mathrm{E}^{\mathrm{N}}$ be defined by

$$
\mathrm{T}^{*}:\left(\mathrm{x}_{1}, \mathrm{x}_{2}, \cdots, \mathrm{x}_{\mathrm{N}}\right) \mapsto(\mathrm{T} x, \mathrm{~T} x, \cdots, \mathrm{T} x)
$$

for all $x=\left(x_{1}, x_{2}, \cdots, x_{N}\right) \in E^{N}$. In this case, we have that

$$
\begin{aligned}
\mathrm{D}_{\mathrm{T}^{*} x, T^{*} y}(\mathrm{t}) & =\mathrm{P}_{\mathrm{T}}\left(\mathrm{F}_{\mathrm{T} x, T y}(\mathrm{t}), \mathrm{F}_{\mathrm{T} x, T y}(\mathrm{t}), \ldots, \mathrm{F}_{\mathrm{T} x, T y}(\mathrm{t})\right) \\
& =\mathrm{F}_{\mathrm{T} x, T y}(\mathrm{t}) \\
& \geqslant \mathrm{P}_{\mathrm{T}}\left(\mathrm{F}_{\mathrm{x}_{1}, y_{1}}\left(\frac{\mathrm{t}}{\mathrm{h}}\right), \mathrm{F}_{\mathrm{x}_{2}, y_{2}}\left(\frac{\mathrm{t}}{\mathrm{h}}\right), \cdots, \mathrm{F}_{\mathrm{x}_{\mathrm{N}, y_{N}}}\left(\frac{\mathrm{t}}{\mathrm{h}}\right)\right) \\
& =\mathrm{D}_{x, y}\left(\frac{\mathrm{t}}{\mathrm{h}}\right)
\end{aligned}
$$

for all $x=\left(x_{1}, x_{2}, \cdots, x_{N}\right) \in E^{N}, y=\left(y_{1}, y_{2}, \cdots, y_{N}\right) \in E^{N}$. By using Theorem 2.9, $T^{*}$ has a unique fixed point $x^{*} \in E^{N}$. That is, there exists a unique $x^{*}=\left(x_{1}^{*}, x_{2}^{*}, \cdots, x_{N}^{*}\right) \in E^{N}$ such that

$$
\begin{aligned}
\mathrm{T}^{*} x^{*} & =\left(\mathrm{T} x^{*}, \mathrm{~T} x^{*}, \cdots, \mathrm{T} x^{*}\right) \\
& =\left(x_{1}^{*}, x_{2}^{*}, \cdots, x_{\mathrm{N}}^{*}\right) .
\end{aligned}
$$

This implies that $x_{1}^{*}=x_{2}^{*}=\cdots=x_{N}^{*}$ and $x_{1}^{*}=T\left(x_{1}^{*}, x_{1}^{*}, \cdots, x_{1}^{*}\right)$, hence $T$ has a unique multivariate fixed point $x_{1}^{*}$. This completes the proof.

From Example 2.2 and Example 2.3, we can get the following results.

Corollary 2.12. Let $(\mathrm{E}, \mathrm{F}, \mathrm{T})$ be a complete Menger probabilistic metric space with a continuous T-norm

$$
\mathrm{T}(\mathrm{a}, \mathrm{b})=\max \{\mathrm{a}+\mathrm{b}-1,0\} .
$$

Let $\mathrm{T}: \mathrm{E}^{\mathrm{N}} \rightarrow \mathrm{E}$ be an $\mathrm{N}$-variables mapping satisfying the following condition:

$$
F_{T x, T y}(t) \geqslant \frac{1}{N}\left(F_{x_{1}, y_{1}}\left(\frac{t}{h}\right)+F_{x_{2}, y_{2}}\left(\frac{t}{h}\right)+\cdots+F_{x_{N}, y_{N}}\left(\frac{t}{h}\right)\right)
$$

for every

$$
x=\left(x_{1}, x_{2}, \cdots, x_{N}\right) \in X^{N}, \quad y=\left(y_{1}, y_{2}, \cdots, y_{N}\right) \in X^{N},
$$

and $\mathrm{t}>0$, where $\mathrm{h} \in(0,1)$ is a constant. Assume there exists $\mathrm{p}_{0} \in \mathrm{E}$ such that $\sup \left\{\mathrm{G}_{\mathrm{p}_{0}}(\mathrm{t}): \mathrm{t} \in \mathrm{R}\right\}=1$, where

$$
\begin{aligned}
\mathrm{G}_{\mathrm{p}_{0}}(\mathrm{t}) & =\inf \left\{\mathrm{F}_{\mathrm{p}_{0}, \mathrm{p}_{\mathrm{n}}}(\mathrm{t})\right\}, \mathrm{n}=1,2,3, \cdots, \mathrm{p}_{1}=\mathrm{T}\left(\mathrm{p}_{0}, \mathrm{p}_{0}, \cdots, \mathrm{p}_{0}\right), \\
\mathrm{p}_{2} & =\mathrm{T}\left(\mathrm{p}_{1}, \mathrm{p}_{1}, \cdots, \mathrm{p}_{1}\right), \\
\mathrm{p}_{3} & =\mathrm{T}\left(\mathrm{p}_{2}, \mathrm{p}_{2}, \cdots, \mathrm{p}_{2}\right), \\
& \vdots \\
\mathrm{p}_{\mathrm{n}} & =\mathrm{T}\left(\mathrm{p}_{\mathrm{n}-1}, \mathrm{p}_{\mathrm{n}-1}, \cdots, \mathrm{p}_{\mathrm{n}-1}\right),
\end{aligned}
$$

Then Thas a unique multivariate fixed point.

Corollary 2.13. Let $(\mathrm{E}, \mathrm{F}, \mathrm{T})$ be a complete Menger probabilistic metric space with a continuous $\mathrm{T}-n \mathrm{nom} \mathrm{T}(\mathrm{a}, \mathrm{b})=$ $\max \{\mathrm{a}+\mathrm{b}-1,0\}$. Let $\mathrm{T}: \mathrm{E}^{\mathrm{N}} \rightarrow \mathrm{E}$ be an $\mathrm{N}$-variables mapping satisfying the following condition:

$$
F_{T x, T y}(t) \geqslant \lambda_{1} F_{x_{1}, y_{1}}\left(\frac{t}{h}\right)+\lambda_{2} F_{x_{2}, y_{2}}\left(\frac{t}{h}\right)+\cdots+\lambda_{N} F_{x_{N}, y_{N}}\left(\frac{t}{h}\right),
$$

for every

$$
x=\left(x_{1}, x_{2}, \cdots, x_{N}\right) \in X^{N}, \quad y=\left(y_{1}, y_{2}, \cdots, y_{N}\right) \in X^{N},
$$


and $\mathrm{t}>0$, where $\mathrm{h} \in(0,1)$ is a constant and $\lambda_{i} \in(0,1), i=1,2,3, \cdots, \mathrm{N}$ are constants with $\sum_{i=1}^{\mathrm{N}} \lambda_{i}=1$. Assume there exists $\mathrm{p}_{0} \in \mathrm{E}$ such that $\sup \left\{\mathrm{G}_{\mathrm{p}_{0}}(\mathrm{t}): \mathrm{t} \in \mathrm{R}\right\}=1$, where

$$
\begin{aligned}
\mathrm{G}_{\mathrm{p}_{0}}(\mathrm{t}) & =\inf \left\{\mathrm{F}_{\mathrm{p}_{0}, \mathrm{p}_{\mathrm{n}}}(\mathrm{t})\right\}, \quad \mathrm{n}=1,2,3, \cdots, \\
\mathrm{p}_{1} & =\mathrm{T}\left(\mathrm{p}_{0}, \mathrm{p}_{0}, \cdots, \mathrm{p}_{0}\right), \\
\mathrm{p}_{2} & =\mathrm{T}\left(\mathrm{p}_{1}, \mathrm{p}_{1}, \cdots, \mathrm{p}_{1}\right), \\
\mathrm{p}_{3} & =\mathrm{T}\left(\mathrm{p}_{2}, \mathrm{p}_{2}, \cdots, \mathrm{p}_{2}\right), \\
& \vdots \\
\mathrm{p}_{\mathrm{n}} & =\mathrm{T}\left(\mathrm{p}_{\mathrm{n}-1}, \mathrm{p}_{\mathrm{n}-1}, \cdots, \mathrm{p}_{\mathrm{n}-1}\right),
\end{aligned}
$$

Then T has a unique multivariate fixed point.

The result of Sehgal and Bharucha-Ried [21] was proved by using Picard's iterative sequence method. Therefore, in our results, the multivariate fixed point of T can be also approximated by using Picard's iterative sequence starting any given initial point. We can use the following iterative method to approximate the multivariate fixed point of $\mathrm{T}$.

Iterative sequence 2.14. For any $p_{0} \in E^{N}$, the iterative sequence $\left\{p_{n}\right\} \subset E^{N}$ defined by $p_{n+1}=T^{*} p_{n}, n=$ $0,1,2, \cdots$ converges to a unique fixed point $p^{*}$ of $T^{*}$ in the complete Menger probabilistic metric space $\left(E^{N}, D, T\right)$. Let $p^{*}=\left(p_{1}^{*}, p_{2}^{*}, \cdots, p_{N}^{*}\right)$, from the definition of $T^{*}$, we know that

$$
\begin{aligned}
p^{*} & =\left(p_{1}^{*}, p_{2}^{*}, \cdots, p_{N}^{*}\right) \\
& =T^{*} p^{*} \\
& =\left(T\left(p_{1}^{*}, p_{2}^{*}, \cdots, p_{N}^{*}\right), T\left(p_{1}^{*}, p_{2}^{*}, \cdots, p_{N}^{*}\right), \cdots, T\left(p_{1}^{*}, p_{2}^{*}, \cdots, p_{N}^{*}\right)\right) .
\end{aligned}
$$

This implies that

$$
\mathrm{p}_{1}^{*}=\mathrm{p}_{2}^{*}=\cdots=\mathrm{p}_{\mathrm{N}}^{*}=\mathrm{T}\left(\mathrm{p}_{1}^{*}, \mathrm{p}_{2}^{*}, \cdots, \mathrm{p}_{\mathrm{N}}^{*}\right) .
$$

Hence, we can denote $\mathrm{p}^{*}$ by

$$
p^{*}=(p, p, \cdots, p) .
$$

Let $p_{0}=\left(p_{0,1}, p_{0,2}, \cdots, p_{0, N}\right)$, then the iterative sequence

$$
\begin{aligned}
p_{1} & =\left(T p_{0}, T p_{0}, \cdots, T p_{0}\right) \\
p_{2} & =\left(T p_{1}, T p_{1}, \cdots, T p_{1}\right) \\
p_{3} & =\left(T p_{2}, T p_{2}, \cdots, T p_{2}\right) \\
& \vdots \\
p_{n+1} & =\left(T p_{n}, T p_{n}, \cdots, T p_{n}\right)
\end{aligned}
$$

converges to a unique fixed point $p^{*}$ of $T^{*}$ in the complete Menger probabilistic metric space $\left(E^{N}, D, T\right)$. Since

$$
\begin{aligned}
\mathrm{D}_{p_{n}, p^{*}}(t) & =\mathrm{P}_{\mathrm{T}}\left(\mathrm{F}_{\mathrm{T} p_{\mathrm{n}-1}, \mathrm{p}}(\mathrm{t}), \mathrm{F}_{\mathrm{T} p_{\mathrm{n}-1}, \mathrm{p}}(\mathrm{t}), \cdots, \mathrm{F}_{\mathrm{T} p_{\mathfrak{n}-1}, \mathrm{p}}(\mathrm{t})\right) \\
& =\mathrm{F}_{\mathrm{T} p_{\mathfrak{n}-1, p}, \mathrm{p}}(\mathrm{t})
\end{aligned}
$$

for all $n=1,2,3, \cdots$. Therefore, the sequence $\left\{T p_{n}\right\}$ converges to a unique multivariate fixed point $p$ of $T$ in the complete Menger probabilistic metric space $(E, F, T)$.

\section{Acknowledgment}

This project is supported by the major project of Hebei North University under grant No. ZD201304. 


\section{References}

[1] S.-S. Chang, Y. J. Cho, S.-M. Kang, Nonlinear Operator Theory in Probabilitic Metric Spaces, Nova Publisher, New York, (2001). 1

[2] S. Chauhan, S. Bhatnagar, S. Radenović, Common fixed point theorems for weakly compatible mappings in fuzzy metric spaces, Matematiche, 68 (2013), 87-98. 1

[3] B. Choudhury, K. Das, A new contraction principle in Menger spaces, Acta Math. Sin., 24 (2008), 1379-1386.

[4] B. S. Choudhury, K. Das, A coincidence point result in Menger spaces using a control function, Chaos Solitons \& Fractals, 42 (2009), 3058-3063. 1

[5] B. S. Choudhury, K. Das, P. N. Dutta, A fixed point result in Menger spaces using a real function, Acta Math. Hungar., 122 (2009), 203-216.

[6] S. Chauhan, S. Dalal, W. Sintunavarat, J. Vujaković, Common property (E.A) and existence of fixed points in Menger spaces, J. Inequal. Appl., 2014 (2014), 14 pages. 1

[7] S. Chauhan, M. Imdad, C. Vetro, W. Sintunavarat, Hybrid coincidence and common fixed point theorems in Menger probabilistic metric spaces under a strict contractive condition with an application, Appl. Math. Comput., 239 (2014), 422-433. 1

[8] S. Chauhan, S. Radenović, M. Imdad, C. Vetro, Some integral type fixed point theorems in non-Archimedean Menger PM-spaces with common property (E.A) and application of functional equations in dynamic programming, Rev. R. Acad. Cienc. Exactas Fs. Nat. Ser. A Math., 108 (2014), 795-810.

[9] L. B. Ćirć, On fixed point of generalized contractions on probabilistic metric spaces, Publ. Inst. Math., 18 (1975), 71-78.

[10] P. N. Dutta, B. S. Choudhury, K. Das, Some fixed point results in Menger spaces using a control function, Surv. Math. Appl., 4 (2009), 41-52.

[11] L. Gajić, V. Rakoćević, Pair of non-self-mappings and common fixed points, Appl. Math. Comput., 187 (2007), $999-1006$. 1

[12] O. Hadzic, E. Pap, Fixed Point Theory in Probabilistic Metric Spaces, Kluwer Academic, Dordrecht, (2001). 1

[13] M. S. Khan, M. Swaleh, S. Sessa, Fixed points theorems by altering distances between the points, Bull. Aust. Math. Soc., 30 (1984), 1-9. 1

[14] M. A. Kutbi, D. Gopal, C. Vetro, W. Sintunavarat, Further generalization of fixed point theorems in Menger PM-spaces, Fixed Point Theory and Appl., 2015 (2015), 10 pages. 1

[15] H. Lee, S. Kim, Multivariate coupled fixed point theorems on ordered partial metric spaces, J. Korean Math. Soc., 51 (2014), 1189-1207. 1

[16] K. Menger, Statistical metrics, Proc. Nat. Acad. Sci. U. S. A., 28 (1942), 535-537. 1

[17] K. Menger, Probabilistic Geometry, Proc. Nat. Acad. Sci. U. S. A., 37 (1951), 226-229. 1

[18] D. Miheț, Altering distances in probabilistic Menger spaces, Nonlinear Anal., 71 (2009), 2734-2738. 1

[19] B. Schweizer, A. Sklar, Probabilistic Metric Spaces, North-Holland Publishing Co., New York, (1983). 1

[20] V. Sehgal, Some Fixed Point Theorems in Functional Analysis and Probability, Ph.D. Dissertation, Wayne State University, Michigan, (1966). 1

[21] V. Sehgal, A. Bharucha-Reid, Fixed point of contraction mappings in PM-spaces, Math. Syst. Theory., 6 (1972), 97-102. $1,2,2.8,2.9,2,2$

[22] Y. Su, W. Gao, J. Yao, Generalized contraction mapping principle and generalized best proximity point theorems in probabilistic metric spaces, Fixed Point Theory and Appl., 2015 (2015), 20 pages. 1

[23] Y. Su, A. Petrusel, J.-C. Yao, Multivariate fixed point theorems for contractions and nonexpansive mappings with applications, Fixed Point Theory and Appl., 2016 (2016), 19 pages. 1

[24] Y. Su, J. Zhang, Fixed point and best proximity point theorems for contractions in new class of probabilistic metric spaces, Fixed Point Theory and Appl., 2014 (2014), 15 pages. 1

[25] T. Van An, N. Van Dung, Z. Kadelburg, S. Radenović, Various generalizations of metric spaces and fixed point theorems, Rev. R. Acad. Cienc. Exactas Fs. Nat. Ser. A Math., 109 (2015), 175-198. 1 